\title{
Spiropyrazolines From Tandem Reaction of Azides and Alkyl Vinyl Ketones
}

\author{
Chia-Hsi Yang*, Lang-Tone Lee and Jun-Hao Yang \\ Department of Chemistry, Chung-Yuan Christian University, Chung-Li, Taiwan, R.O.C. \\ Yu Wang and Gene-Hsiang Lee \\ Department of Chemistry,National Taiwan University, Taipei, Taiwan, R.O.C.
}

\begin{abstract}
Spiropyrazolines are obtained from the the tandem reactions of one molecule of azide and three molecules of alkyl vinyl ketone. There are two types of structures in these products: One of them has an intramolecular hydrogen bond between the hydroxyl proton and the acetyl oxygen, while the other has none.
\end{abstract}

Alkyl azides and alkenes carrying electron-withdrawing groups, such as acrylate and acrylonitrile, undergo intermolecular 1,3-dipolar cycloaddition, isomerization of the resulting $\Delta^{2}$-triazolines to diazo compounds and subsequently another intermolecular 1,3-dipolar cycloaddition to give $\Delta^{2}$-pyrazolines(1) was reported by L'abbe et al. (Scheme 1, pathway a) ${ }^{1}$

However, when allyl azide replaces alkyl azide, the reaction of azide with alkenes carrying electron-withdrawing groups gives different results. If the alkene is maintained at a low concentration during the reaction, a good yield of 2,3,7-triazabicyclo-[3.3.0]oct-2-enes(2), which should be the product from intermolecular 1,3dipolar cycloaddition, followed by isomerization to a diazo compound, an intramolecular 1,3-dipolar cycloaddition and, in some cases, a Michael addition (Scheme 1, pathway b), are obtained.2

Maintaining the alkene at a low concentration during the reaction is vital to obtaining a good yield of 2 . If the concentration of alkenes, such as acrylate and acrylonitrile, is comparable to that of allyl azide, not only $\underline{2}$ but also $\underline{1}$, which is the minor by-product following pathway $a$, is obtained.

When allyl azide reacted with methyl vinyl ketone(MVK), which was kept at a low concentration during the reaction, a good yield of $\underline{\mathbf{2}}$ was obtained as expected. However, if the high concentration of MVK was comparable to that of allyl azide, not only the major product $\underline{2}$ but also an unexpected minor product, which apparently is not a product with structure 1 , was obtained. This new product, which should be an annulation product of one molecule of allyl azide and three molecules of methyl vinyl ketone ( [1+3] product) was evident from the mass spectrum, ${ }^{1} \mathbf{H}$ and ${ }^{13} \mathbf{C}$ NMR spectra. That the double bond of the allyl group is intact indicates that no intra- 
Scheme 1.

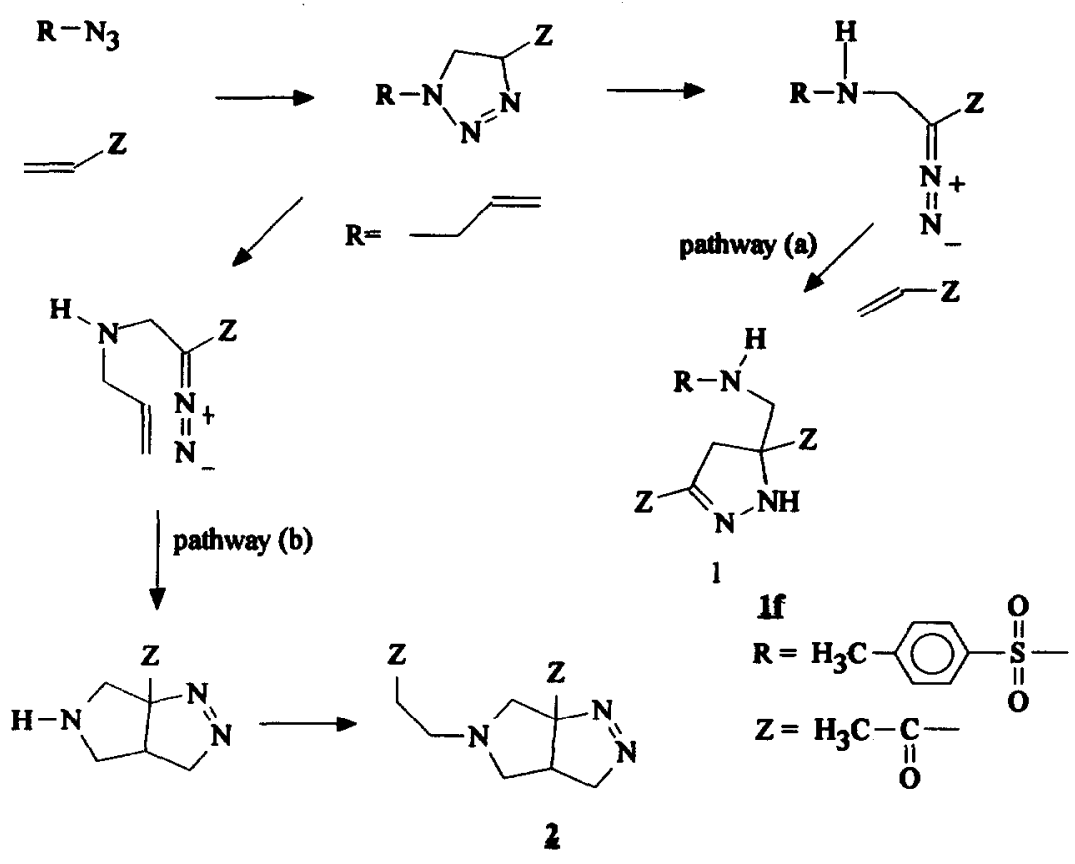

molecular dipolar cycloaddition was involved. With the help of 2-D C-H COSY NMR spectra, we were able to assign its structure as $3 \mathrm{a}$. However, for confirming the assignment, an $x$-ray diffraction study on a single crystal of $3 \mathrm{a}$ was carried out. The result confirms that the assignment is correct. 3a , a spiro compound consisting of $\Delta^{2}$ pyrazoline and piperidine, is a heterocyclic compound with a new skeleton according to our literature survey ${ }^{3}$. Similarly, minor by-products $3 \mathbf{b}, 3 \mathrm{~g}$ and $3 \mathbf{i}$ were obtained from the reaction of isobutenyl azide with methyl vinyl ketone, the reaction of allyl azide with ethyl vinyl ketone and the reaction of 2-butenyl azide with ethyl vinyl ketone respectively.

Since the double bond of the allyl group is not involved in the reaction for synthesis of $\underline{3 a}, \underline{3 b}, 3 \mathrm{~g}$ and $\underline{3 \mathrm{i}}$, it is expected that the reaction of alkyl azide with 
<smiles>[R2]C(=O)C1=NNC2(C1)CN([R])CC(C([R2])=O)C2([R2])O</smiles>

3

\begin{tabular}{|l|l|l|l|}
\hline & \multicolumn{1}{|c|}{$\mathrm{R}_{1}$} & $\mathrm{R}_{2}$ & type \\
\hline$\underline{\mathbf{3 a}}$ & $\mathrm{H}_{2} \mathrm{C}=\mathrm{CHCH}_{2}$ & $\mathrm{CH}_{3}$ & NHB \\
\hline$\underline{\mathbf{3 b}}$ & $\mathrm{H}_{2} \mathrm{C}=\mathrm{C}\left(\mathrm{CH}_{3}\right)-\mathrm{CH}_{2}$ & $\mathrm{CH}_{3}$ & $\mathbf{H B}$ \\
\hline$\underline{\underline{3 c}}$ & $\mathrm{CH}_{3} \mathrm{CH}_{2} \mathrm{CH}_{2}$ & $\mathrm{CH}_{3}$ & HB \\
\hline$\underline{\underline{3 \mathbf{d}}}$ & benzyl & $\mathrm{CH}_{3}$ & HB \\
\hline$\underline{\underline{3 f}}$ & $\mathrm{CH}_{3} \mathrm{CH}_{2} \mathrm{CH}_{2}$ & $\mathrm{C}_{2} \mathrm{H}_{5}$ & NHB \\
\hline$\underline{\underline{3 g}}$ & toluenesulfonyl & $\mathrm{CH}_{3}$ & HB \\
\hline$\underline{\mathbf{3 h}}$ & $\mathrm{H}_{2} \mathrm{C}=\mathrm{CHCH}_{2}$ & $\mathrm{C}_{2} \mathrm{H}_{5}$ & NHB \\
\hline$\underline{3 \mathbf{i}}$ & $\mathrm{H}_{2} \mathrm{C}=\mathrm{CHCH} \mathrm{CH}_{2}$ & $\mathrm{CH}_{3}$ & NHB \\
\hline
\end{tabular}

methyl vinyl ketone or ethyl vinyl ketone, in the absence of the competitive pathway $\mathbf{b}$, may give a much higher yield of product with structure 3 . Therefore, the reaction of propyl azide with methyl vinyl ketone in tetrahydrofuran was studied. The products of the reaction were complicated. Nevertheless, a major product $3 \mathbf{c}$, a [1+3] product, in a yield of $54 \%$ as observed by a ${ }^{1} \mathrm{H}$ NMR spectrum and an isolated yield of $32 \%$ was obtained. Similarly, $\underline{3 d}$, also a $[1+3]$ product was obtained from the reaction of benzyl azide with methyl vinyl ketone. The ${ }^{1} \mathbf{H}$ and ${ }^{13} \mathrm{C}$ NMR spectra of these compounds are consistent with the structures of $\underline{\mathbf{3 c}}$ and $\underline{\mathbf{3 d}}$, spiro compounds consisting of $\Delta^{2}$-pyrazoline and piperidine. However, a careful comparision of the NMR spectra of $3 \mathrm{c}$ and $\underline{\mathbf{3 d}}$ with that of $\underline{3 a}$ shows that there are several differences between them. In $3 \mathrm{a}$, a hydroxyl proton at $\delta 2.58$ was observed in the ${ }^{1} \mathrm{H}$ NMR spectra, whereas, this hydroxyl proton shifts to $\delta 4.02$ in $3 \mathrm{c}$ and $3 \mathrm{~d}$. In the ${ }^{13 \mathrm{C}} \mathrm{NMR}$ spectra, the chemical shift differences between the corresponding carbons in $\underline{3 \mathrm{c}}$ and $\underline{\mathbf{3 d}}$ are less than $0.2 \mathrm{ppm}$. But differences as large as $4.5 \mathrm{ppm}$ were observed between the corresponding carbons in $\underline{3 \mathrm{c}}$ and $\mathbf{3 a}$. Therefore, there must be differences in structures between these two series of compounds. For confirming the assignment of the 
structure, and at the same time finding an explanation of the differences in the NMR spectra, a single crystal $x$-ray diffraction study on $3 \mathrm{~d}$ was carried out. The result confirmed that the product, as $\mathbf{3 a}$, is a spiro compound consisting of 2-pyrazoline and piperidine, however, there are, indeed, differences between the structures of $\mathbf{3 a}$ and $\underline{\mathbf{3 d}}$. Both piperidine rings in $3 \mathrm{a}$ (NHB type) and 3d (HB type)are in chair form. However, their stereochemistries are different, relative to the pyrazoline ring. The piperidine nitrogen $\left(\mathrm{N}^{3}\right)$ is trans- and gauche to pyrazoline nitrogen $\left(\mathrm{N}^{\mathrm{l}}\right)$ in $\underline{\mathbf{3 a}}$ and $\underline{\mathbf{3 d}}$, respectively.
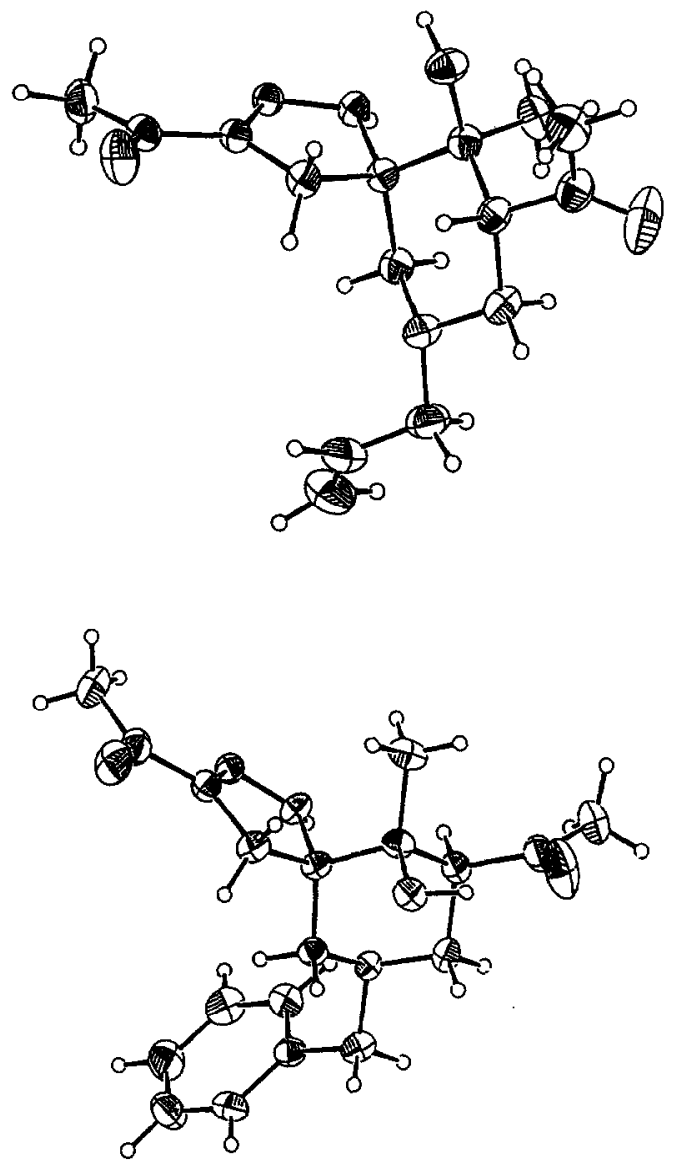

Figures : Molecular structures of $\underline{\mathbf{3 a}}$ and $\underline{\mathbf{3 d}}$ 
The hydroxyl group in $\mathbf{3 d}$ is in axial conformation and intramolecularly hydrogenbonded to the neighboring equatorial acetyl oxygen. The corresponding hydroxyl group in $\underline{3 a}$ is in equatorial conformation and has no hydrogen bond. These differences account for the large differences in the chemical shifts in ${ }^{1} \mathbf{H}$ and ${ }^{13} \mathrm{C}$ NMR spectra of $\underline{3 \mathrm{a}}$ and $\underline{3 \mathrm{~d}}$.

As a further study on the scope of the reaction, the reaction of propyl azide with ethyl vinyl ketone, and toluenesulfonyl azide with methyl vinyl ketone were studied. In both cases, $[1+3]$ products were obtained. $\underline{3 e}$ is a NHB type compound and $\underline{3 f}$ is a HB type compound. It is known that pathway $b$ is not followed in the reaction of 3butenyl azide with alkene carrying an electron-withdrawing group ${ }^{2}$, therefore, $3 \mathbf{h},[1+$ 3] product, is obtained as the major product as expected from the reaction of 3butenyl azide with methyl vinyl ketone.<smiles>CC(=O)C1=NN(I)C(CN(C)C)(C(C)=O)C1</smiles>

$\underline{1}$<smiles>[Z]C(=O)OC(C)=O</smiles>

$\underline{3}$

Compound $\underline{3}$ must be a further reaction product from $\underline{1}$, as is evident from the presence of $\underline{1}$ as a partial structure in $\underline{3}$. A trivial explanation is that a Michael addition of amino nitrogen of 1 to methyl vinyl ketone gives $\beta$-aminoketone, which undergoes an Aldol condensation to give product 3. Actually, if can be isolated as the product in the reaction of toluenesulfonyl azide with methyl vinyl ketone.

$\underline{3 c}, 3 \mathrm{~d}, 3 \mathrm{e}$ and $3 \mathrm{f}$ are the major products in the reaction, thorough only in a yield of around $30 \%$. This moderate yield is understandable, since there are five steps from the starting materials to the final product and several side reactions are possible. Yet, a yield of $30 \%$ is still equivalent to $75 \%$ average yield/each step. A multiplecomponents annulation has been applied to synthesize complex, non-polymeric compound. 4-6 $^{-6}$ the present case, a complicated heterocyclic compound could be obtained from simple starting materials in one pot.

The temperature of the reaction was kept at room temperature. At a lower temperature the reaction time would be unbearably long and at higher temperature, say $50{ }^{\circ} \mathrm{C}$, the reaction becomes more complicated, and the yield of $[1+3]$ product diminishes.

In the reaction of azide with alkyl vinyl ketone, either a NHB type or a HB type product, but never both, was isolated in each reaction. In the hope of obtaining the other stereoisomer that might also be present in the reaction, careful separation had 
been tried repeatedly without success. However, we were not able to rationalize the generalization of the stereospecificity of the reaction.

\section{Experimental}

Melting points were determined on a Yanagimoto micromelting point apparatus and are reported uncorrected. The ${ }^{1} \mathrm{H}$ NMR and ${ }^{13} \mathrm{C}$ NMR spectra were determined on a Brucker ACE-200 MHz FT-NMR spectrometer using TMS and $\mathrm{CDCl}_{3}$ as internal standards, respectively. 2D NMR spectra were obtained by a Ace-300 $\mathrm{MHz}$ FT-NMR spectrometer using INVDR2LP program?. The mass spectra were obtained on a JEOL JMS-SX/SX 102A spectrometer operating at 12ev. The elemental composition of compounds were determined by a JOEL JMS-SX/SX 102A high resolution mass spectrometer.

General procedure for the reaction of azide and methyl vinyl ketone: To 0.03 mole of azide dissolved in $20 \mathrm{ml}$ tetrahydrofuran, 0.1 mole of alkyl vinyl ketone was added dropwise. The reaction mixture was then stirred at room temperature for 24 hours. After evaporation of the solvent, the $[1+3]$ product was obtained from column chromatography of the brown viscose crude product using silica gel as the stationary phase and n-hexane: ether $=5: 2$ as the eluent.

3,9-Diacetyl-10-hydroxy-10-methyl-7-(2-propenyl)-1,2,7-triazaspiro[4.5]dec-2ene(3a) ${ }^{8}$ Yellow crystal, m.p. $190-192{ }^{\circ} \mathrm{C}\left(\mathrm{Et}_{2} \mathrm{O}\right)$; ${ }^{1} \mathrm{H}$ NMR $\delta 1.04\left(\mathrm{~s}, 3 \mathrm{H}, \mathrm{CH}_{3}\right)$, 2.00, 2.61(AB system, J=11.7 Hz, 2H, $\mathrm{CH}_{2}$ ), 2.25(s, 3H, $\mathrm{CH}_{3}$ ), 2.31, 2.73, 2.79(ABX, $\left.\mathrm{J}_{\mathrm{AX}}=12.0 \mathrm{~Hz}, \mathrm{~J}_{\mathrm{BX}}=11.0 \mathrm{~Hz}, \mathrm{~J}_{\mathrm{AB}}=4.0 \mathrm{~Hz}, 3 \mathrm{H}, \mathrm{CHCH}_{2}\right), 2.38\left(\mathrm{~s}, 3 \mathrm{H}, \mathrm{CH}_{3}\right), 2.42$, 2.98(AB system, $\mathrm{J}=17.4 \mathrm{~Hz}, 2 \mathrm{H}, \mathrm{CH}_{2}$ ), 2.58(s, $\left.\mathrm{OH}\right), 2.95-3.00\left(\mathrm{~m}, 2 \mathrm{H}, \mathrm{CH}_{2}\right)$, 5.075.16(m, 2H, vinylic $\left.\mathrm{CH}_{2}\right), 5.63-5.76(\mathrm{~m}, 1 \mathrm{H}$, vinylic $\mathrm{CH}), 6.83(\mathrm{~s}, 1 \mathrm{H}, \mathrm{NH}) ;{ }^{13} \mathrm{C}$ NMR $\delta$ 17.5 $\left(\mathrm{CH}_{3}\right), 25.3\left(\mathrm{CH}_{3}\right), 32.9\left(\mathrm{CH}_{3}\right), 34.2\left(\mathrm{CH}_{2}\right), 51.7\left(\mathrm{CH}_{2}\right), 57.5\left(\mathrm{CH}_{2}\right), 58.1(\mathrm{CH})$, 60.3( $\left(\mathrm{CH}_{2}\right), 71.8(\mathrm{C}), 73.7(\mathrm{C}), 118.6\left(\mathrm{CH}_{2}\right), 134.1(\mathrm{CH}), 153.9(\mathrm{CN}), 194.7(\mathrm{CO}), 209.1$

(CO); MS m/z(\%) 293(M+1 13), 250 (5), 193(8), 140(27), 96(25), 84(100), 70(63); HRMS: Calcd for $\mathrm{C}_{15} \mathrm{H}_{23} \mathrm{~N}_{3} \mathrm{O}_{3}: 293.1739$, Found: 293.1741. X-ray crystallographic structure determination of $3 \mathrm{a}$ : Yellow single crystal suitable for the collection of $\mathrm{X}$ ray diffraction data were obtained by recrystallization from ether. A crystal (dimension $0.4 \times 0.6 \times 0.6 \mathrm{~mm}$ ) was selected for data collection and mounted on an Enraf-Nonius CAD-4 automated fourcircle diffractometer. The crystal was found to be monoclinic, and unit cell parameters and the orientation matrix were obtained from 25 reflections in the range $18^{\circ}<2 \theta<25^{\circ}$. Data collection was accomplished by using $\theta / 2 \theta$ scan technique with $2.06-8.24^{\circ} / \mathrm{min}$ : space group $\mathrm{C} 2 / \mathrm{c} ; \mathrm{a}=2600.4(6) \AA ; \mathrm{b}=716.82(17) \AA$; $c=1862.1(5) \AA ; \beta=109.13(2)^{\circ} ; \mathrm{V}=3279 \AA^{3} ; \underline{d}$ calcd $=1.19 \mathrm{~g} / \mathrm{cm}^{3}(\mathrm{Z}=4)$;radiation Mo- $\mathrm{K}_{\alpha} ; 2 \theta$ limits (max) $50.0^{\circ}$; total reflections scanned $2876 ; 2876$ unique reflections, 1846 "observed " reflections with $\mathrm{I}>2 \sigma(\mathrm{I}) ; \mathrm{R}=0.045 ; R_{\mathrm{W}}=0.053 ; 191$ refined 
parameters. Crystallographic computations were carried out on a Micro Vax III computer using the NRCC-SDD-VAX structure determining package?. The structure was solved by direct methods. The non-hydrogen atoms were refined anisotropically. The hydrogen atoms were localized by difference electron density determination. List of positional and anisotropic thermal parameters of nonhydrogen atoms, positional and thermal parameters of hydrogen atoms, bond distances and bond angles are available upon request.

3,9-Diacetyl-10-hydroxy-10-methyl-7-(2-methyl-2-propenyl)-1,2,7-triazaspiro[4.5]dec-2-ene(3b). ${ }^{8}$ White crystal, m.p. $148-150{ }^{\circ} \mathrm{C}\left(\mathrm{Et}_{2} \mathrm{O}\right)$; ${ }^{1} \mathrm{H}$ NMR $\delta 1.13(\mathrm{~s}, 3 \mathrm{H}$, $\mathrm{CH}_{3}$ ), 1.69(s, 3H, $\mathrm{CH}_{3}$ ), 2.23(s, 3H, $\mathrm{CH}_{3}$ ), 2.32, 3.02(AB system, $2 \mathrm{H}, \mathrm{J}=18.6 \mathrm{~Hz}$, $\mathrm{CH}_{2}$ ), 2.35, 2.43(AB system, $\left.2 \mathrm{H}, \mathrm{J}=11.0 \mathrm{~Hz}, \mathrm{CH}_{2}\right), 2.42,2.73,2.91\left(\mathrm{ABX}, \mathrm{J}_{\mathrm{AX}}=11.4\right.$ $\left.\mathrm{Hz}, \mathrm{J}_{\mathrm{BX}}=11.0 \mathrm{~Hz}, \mathrm{~J}_{\mathrm{AB}}=4.0 \mathrm{~Hz}, 3 \mathrm{H}, \mathrm{CHCH}_{2}\right), 2.36\left(\mathrm{~s}, 3 \mathrm{H}, \mathrm{CH}_{3}\right), 2.86\left(\mathrm{~s}, 2 \mathrm{H}, \mathrm{CH}_{2}\right)$, $4.04(\mathrm{~s}, \mathrm{OH}), 4.84\left(\mathrm{~s}, 2 \mathrm{H}, \mathrm{C}=\mathrm{CH}_{2}\right), 6.6(\mathrm{~s}, \mathrm{NH}) ;{ }^{13} \mathrm{C} \mathrm{NMR} \delta 20.7\left(\mathrm{CH}_{3}\right), 22.0\left(\mathrm{CH}_{3}\right)$, 25.3( $\left(\mathrm{CH}_{3}\right), 31.9\left(\mathrm{CH}_{3}\right), 34.5\left(\mathrm{CH}_{2}\right), 50.8\left(\mathrm{CH}_{2}\right), 54.5(\mathrm{CH}), 57.4\left(\mathrm{CH}_{2}\right), 64.2\left(\mathrm{CH}_{2}\right)$, 70.3(C), 71.9 (C), 113.9( $\left.\mathrm{CH}_{2}\right), 141.4(\mathrm{C}), 151.0(\mathrm{C}), 194.6(\mathrm{C}), 213.4(\mathrm{C}) ; \mathrm{MS} \mathrm{m} / \mathrm{e}$ (EIMS, \%) $307\left(\mathrm{M}^{+}, 69\right), 124(14), 110(17), 98(100), 84$ (56), 71(17), 55(36); HRMS: Calcd for $\mathrm{C}_{16} \mathrm{H}_{25} \mathrm{~N}_{3} \mathrm{O}_{3}$ : 307.1890307 .1897 .

3,9-Diacetyl-10-hydroxy-10-methyl-7-propyl-1,2,7-triazaspiro[4.5] dec-2-ene(3c). $32 \%$ yield. Yellow liquid; ${ }^{1} \mathrm{H}$ NMR $\delta 0.82\left(\mathrm{t}, \mathrm{J}=7.2 \mathrm{HZ}, 3 \mathrm{H}, \mathrm{CH}_{3}\right), 1.09\left(\mathrm{~s}, 3 \mathrm{H}, \mathrm{CH}_{3}\right.$ ), 1.29 - $1.49\left(\mathrm{~m}, 2 \mathrm{H}, \mathrm{CH}_{2}\right), 2.20-2.45\left(\mathrm{~m}, 2 \mathrm{H}, \mathrm{CH}_{2}\right), 2.21\left(\mathrm{~s}, 3 \mathrm{H}, \mathrm{CH}_{3}\right), 2.30,2.72,2.92$ $\left(\mathrm{AMX}, 3 \mathrm{H}, \mathrm{J}_{\mathrm{AX}}=12.0 \mathrm{~Hz}, \mathrm{~J}_{\mathrm{BX}}=11.0 \mathrm{~Hz}, \mathrm{~J}_{\mathrm{AB}}=3.8 \mathrm{~Hz}, \mathrm{CHCH}_{2}\right.$ ), 2.33(s, 3H, $\mathrm{CH}_{3}$ ), 2.37, 2.98(AB system, $\mathrm{J}=18.8 \mathrm{~Hz}, 2 \mathrm{H}, \mathrm{CH}_{2}$ ) $2.30,2.45$ ( $\mathrm{AB}$ system, $\mathrm{J}=11.4 \mathrm{~Hz}, 2 \mathrm{H}$, $\left.\mathrm{CH}_{2}\right), 4.02(\mathrm{~s}, \mathrm{OH}), 6.8(\mathrm{~s}, \mathrm{NH}) ;{ }^{13} \mathrm{C} \mathrm{NMR} \delta 11.5\left(\mathrm{CH}_{3}\right), 19.6\left(\mathrm{CH}_{2}\right), 21.9\left(\mathrm{CH}_{3}\right)$, 25.1 $\left(\mathrm{CH}_{3}\right), 31.7\left(\mathrm{CH}_{3}\right), 34.4\left(\mathrm{CH}_{2}\right), 50.9\left(\mathrm{CH}_{2}\right), 54.4(\mathrm{CH}), 57.4\left(\mathrm{CH}_{2}\right), 59.2\left(\mathrm{CH}_{2}\right)$, 70.2(C), 71.8(C), 150.4(CN), 194.4(CO), 213.2(CO); MS m/z(\%): 295(M+, 54), 266 (24), 194(12), 172(32), 142(58), 86(100), 72(77); HRMS: Calcd for $\mathrm{C}_{15} \mathrm{H}_{25} \mathrm{~N}_{3} \mathrm{O}_{3}$ : 295.1904, Found: 295.1904.

3,9-Diacetyl-7-benzyl-10-hydroxy-10-methyl-1,2,7-triazaspiro[4.5]dec-2ene(3d). $34 \%$ Yield. Yellow crystal. mp. $178-180^{\circ} \mathrm{C}\left(\mathrm{Et}_{2} \mathrm{O}\right)$; ${ }^{1} \mathrm{H}$ NMR $\delta 1.12\left(\mathrm{~s}, 3 \mathrm{H}, \mathrm{CH}_{3}\right)$, 2.20(s, 3H, $\mathrm{CH}_{3}$ ), 2.34(s, 3H, $\left.\mathrm{CH}_{3}\right), 2.37,2.74,2.97\left(\mathrm{AMX}, \mathrm{J}_{\mathrm{AX}}=12.0 \mathrm{~Hz}, \mathrm{~J}_{\mathrm{BX}}=\right.$ $10.8 \mathrm{~Hz}, \mathrm{~J}_{\mathrm{AB}}=3.8 \mathrm{~Hz}, 3 \mathrm{H}, \mathrm{CHCH}_{2}$ ), 2.40,3.03(AB system, $2 \mathrm{H}, \mathrm{J}=18.6 \mathrm{~Hz}, \mathrm{CH}_{2}$ ), $2.43,2.56\left(\mathrm{AB}\right.$ system, $\left.2 \mathrm{H}, \mathrm{J}=11.2 \mathrm{~Hz}, \mathrm{CH}_{2}\right), 3.50\left(\mathrm{~s}, 2 \mathrm{H}\right.$, benzylic $\left.\mathrm{CH}_{2}\right), 4.02(\mathrm{~s}, \mathrm{OH})$, $6.71(\mathrm{~s}, \mathrm{NH}), 7.21-7.35(\mathrm{~m}, 5 \mathrm{H}$, aromatic $\mathrm{H}) ;{ }^{13} \mathrm{C} \mathrm{NMR} 22.0\left(\mathrm{CH}_{3}\right), 25.3\left(\mathrm{CH}_{3}\right)$, 32.0 $\left(\mathrm{CH}_{3}\right), 34.6\left(\mathrm{CH}_{2}\right), 50.6\left(\mathrm{CH}_{2}\right), 54.4(\mathrm{CH}), 57.6\left(\mathrm{CH}_{2}\right), 62.0\left(\mathrm{CH}_{2}\right), \quad 70.2(\mathrm{C})$, $71.9(\mathrm{C}), 127.4(\mathrm{CH}), 128.4(\mathrm{CH}), 128.7(\mathrm{CH}), 137.2(\mathrm{C}), 151.1(\mathrm{CN}), 194.5(\mathrm{CO})$, 213.3(CO); MS m/z(\%): 343(M+1 $\left.\mathrm{M}^{+}, 11\right), 282(10), 220(12), 190(9), 134(88), 120(26)$, 
91 (100), 43(25); HRMS: Caled for $\mathrm{C}_{19} \mathrm{H}_{25} \mathrm{~N}_{3} \mathrm{O}_{3}: 343.1890$, Found: 343.1889. X-ray crystallographic structure determination of $3 \mathrm{~d}$ : The details of $\mathrm{X}$-ray diffraction study are the same as those reported for $3 \mathrm{a}$ if not stated in the following. Yellow single crystal suitable for the collection of $\mathrm{X}$-ray diffraction data were obtained by recrystallization from ether. A crystal (dimension $0.2 \times 0.3 \times 0.6 \mathrm{~mm}$ ) was selected for data collection. The crystal was found to be monoclinic and $P 2_{1}$ space group ; a $=$ 874.0(3) $\AA ; b=1066.0(3) \AA ; c=1056.5(4) \AA ; \beta=111.49(3)^{\circ} ; V=915.9(5) \AA^{3}$; $\underline{\mathrm{d}}_{\mathrm{calcd}}=1.25 \mathrm{~g} / \mathrm{cm}^{3}(\mathrm{Z}=2)$; total reflections scanned $1710 ; 1710$ unique reflections, 1366 "observed" reflections with $I>2 \sigma(I) ; R=0.040 ; R_{w}=0.045 ; 226$ refined parameters.

10-Ethyl-10-hydroxy-3,9-dipropionyl-7-propyl-1,2,7-triazaspiro[4.5]dec-2-ene(3e). $30 \%$ yield. White crystal, m.p. $142-144{ }^{\circ} \mathrm{C}\left(\mathrm{Et}_{2} \mathrm{O}\right)$; ${ }^{1} \mathrm{H} \mathrm{NMR} \delta 0.77(\mathrm{t}, \mathrm{J}=7.4 \mathrm{~Hz}, 3 \mathrm{H}$, $\left.\mathrm{CH}_{3}\right), 0.83\left(\mathrm{t}, \mathrm{J}=7.2 \mathrm{~Hz}, 3 \mathrm{H}, \mathrm{CH}_{3}\right), 1.00\left(\mathrm{t}, \mathrm{J}=7.2 \mathrm{~Hz}, 3 \mathrm{H}, \mathrm{CH}_{3}\right), 1.06(\mathrm{t}, \mathrm{J}=7.4 \mathrm{~Hz}, 3 \mathrm{H}$, $\left.\mathrm{CH}_{3}\right), 1.33,1.78\left(\mathrm{qAB}, \mathrm{J}_{\mathrm{AB}}=14.0 \mathrm{~Hz}, \mathrm{~J}=7.0 \mathrm{~Hz}, 2 \mathrm{H}, \mathrm{CH}_{2}\right), 1.39\left(\mathrm{~m}, 2 \mathrm{H}, \mathrm{CH}_{2}\right), 2.01$, 2.57 (AB system, $\mathrm{J}=11.8 \mathrm{~Hz}, 2 \mathrm{H}, \mathrm{CH}_{2}$ ) 2.25-2.32(m, $2 \mathrm{H}, \mathrm{CH}_{2}$ ), 2.40, 2.96, 3.06(ABX, $\mathrm{J}_{\mathrm{AX}}=12.0 \mathrm{~Hz}, \mathrm{~J}_{\mathrm{BX}}=11.0 \mathrm{~Hz}, \mathrm{~J}_{\mathrm{AB}}=3.8 \mathrm{~Hz}, 3 \mathrm{H}, \mathrm{CHCH}_{2}$ ), 2.45, 3.01(AB system, $\mathrm{J}=17.4$ $\left.\mathrm{Hz}, 2 \mathrm{H}, \mathrm{CH}_{2}\right), 2.48(\mathrm{~s}, \mathrm{OH}), 2.83\left(\mathrm{q}, \mathrm{J}=7.0 \mathrm{~Hz}, 2 \mathrm{H}, \mathrm{CH}_{2}\right), 6.83(\mathrm{~s}, \mathrm{NH}) ;{ }^{13} \mathrm{C} \mathrm{NMR} \delta$ 7.55 $\left(\mathrm{CH}_{3}\right), 8.06\left(\mathrm{CH}_{3}\right), 8.33\left(\mathrm{CH}_{3}\right), 11.67\left(\mathrm{CH}_{3}\right), 20.0\left(\mathrm{CH}_{2}\right), 23.84\left(\mathrm{CH}_{2}\right), 23.84\left(\mathrm{CH}_{2}\right)$, $30.98\left(\mathrm{CH}_{2}\right), \quad 34.78\left(\mathrm{CH}_{2}\right), \quad 38.56\left(\mathrm{CH}_{2}\right), \quad 51.71\left(\mathrm{CH}_{2}\right), \quad 57.32(\mathrm{CH}), \quad 57.64\left(\mathrm{CH}_{2}\right)$, 59.03( $\left.\mathrm{CH}_{2}\right), 73.54(\mathrm{C}), 74.37(\mathrm{C}), 153.51(\mathrm{CN}), 197.79(\mathrm{CO}), 212.35(\mathrm{CO})$; $\mathrm{MS} \mathrm{m} / \mathrm{z}(\%)$ : $337\left(\mathrm{M}^{+}, 5\right), 308(5), 200(10), 156(30), 87(100), 73(78), 58(38)$; HRMS: Calcd for $\mathrm{C}_{18} \mathrm{H}_{31} \mathrm{~N}_{3} \mathrm{O}_{3}: 337.2358$, Found: 337.2370 .

3,9-Diacetyl-10-hydroxy-10-methyl-7-(p-toluenesulfonyl)-1,2,7-triazaspiro-[4.5] dec-2-ene(3f). $36 \%$ yield. White crystal, m.p. $142-144^{\circ} \mathrm{C}\left(\mathrm{Et}_{2} \mathrm{O}\right)$; ${ }^{1} \mathrm{H}$ NMR $\delta 1.13(\mathrm{~s}$, $\left.3 \mathrm{H}, \mathrm{CH}_{3}\right), 1.63\left(\mathrm{~s}, 3 \mathrm{H}, \mathrm{CH}_{3}\right), 2.33\left(\mathrm{~s}, 3 \mathrm{H}, \mathrm{CH}_{3}\right), 2.39\left(\mathrm{~s}, 3 \mathrm{H}, \mathrm{CH}_{3}\right), 2.40,3.04(\mathrm{AB}$ system, $\left.2 \mathrm{H}, \mathrm{J}=18.8 \mathrm{~Hz}, \mathrm{CH}_{2}\right), 2.55,3.32,3.72\left(\mathrm{ABX}, \mathrm{J}_{\mathrm{AX}}=12.0 \mathrm{~Hz}, \mathrm{~J}_{\mathrm{BX}}=10.8 \mathrm{~Hz}\right.$, $\left.\mathrm{J}_{\mathrm{AB}}=4.0 \mathrm{~Hz}, 3 \mathrm{H}, \mathrm{CHCH}_{2}\right), 2.62,3.08\left(\mathrm{AB}\right.$ system, $\left.\mathrm{J}=12 \mathrm{~Hz}, 2 \mathrm{H}, \mathrm{CH}_{2}\right), 3.76(\mathrm{~s}, \mathrm{OH})$, $6.69(\mathrm{~s}, \mathrm{NH}), 7.32,7.57(\mathrm{AB}$ system, $\mathrm{J}=8.0 \mathrm{~Hz}, 4 \mathrm{H}$, aromatic $\mathrm{H}) ;{ }^{13} \mathrm{C} \mathrm{NMR} \delta$ $21.56\left(\mathrm{CH}_{3}\right), \quad 21.80\left(\mathrm{CH}_{3}\right), \quad 25.42\left(\mathrm{CH}_{3}\right), \quad 32.51\left(\mathrm{CH}_{3}\right), \quad 34.76\left(\mathrm{CH}_{2}\right), \quad 43.88\left(\mathrm{CH}_{2}\right)$, $49.87\left(\mathrm{CH}_{2}\right), 53.11(\mathrm{CH}), 69.61(\mathrm{C}), 71.86(\mathrm{C}), 127.53(\mathrm{CH}), 130.08(\mathrm{CH}), 132.16(\mathrm{C})$, 144.52(C), $150.52(\mathrm{CN}), 194.67(\mathrm{CO}), 212.34(\mathrm{CO}) ; \mathrm{MS} \mathrm{m} / \mathrm{z}(\%): 407\left(\mathrm{M}^{+}, 10\right)$, 252(37), 155(35), 152(90), 123(100), 101(36), 91(81), 71(22), 66 (26); HRMS: Calcd for $\mathrm{C}_{19} \mathrm{H}_{25} \mathrm{~N}_{3} \mathrm{O}_{5} \mathrm{~S}: 407.1509$, Found: 407.1507 .

10-Ethyl-7-(2-propenyl)-3,9-dipropionyl-1,2,7-triazaspiro[4.5]dec-2-ene(3g). ${ }^{8}$ White crystal, m.p. $174-176{ }^{\circ} \mathrm{C}\left(\mathrm{Et}_{2} \mathrm{O}\right)$; ${ }^{1} \mathrm{H} \mathrm{NMR} \delta \quad 0.76\left(\mathrm{t}, \mathrm{J}=7.6 \mathrm{~Hz}, 3 \mathrm{H}, \mathrm{CH}_{3}\right)$, 
$0.98\left(\mathrm{t}, \mathrm{J}=7.4 \mathrm{~Hz}, 3 \mathrm{H}, \mathrm{CH}_{3}\right), 1.05\left(\mathrm{t}, \mathrm{J}=7.4 \mathrm{~Hz}, 3 \mathrm{H}, \mathrm{CH}_{3}\right), 1.29-1.40,1.68-1.84(\mathrm{~m}, 2 \mathrm{H}$, $\mathrm{CH}_{2}$ ), 1.97, 2.65( $\mathrm{AB}$ system, $\left.\mathrm{J}=12.0 \mathrm{~Hz}, 2 \mathrm{H}, \mathrm{CH}_{2}\right), 2.50,2.97,3.06\left(\mathrm{ABX}, \mathrm{J}_{\mathrm{AX}}=11.8\right.$ $\left.\mathrm{Hz}, \mathrm{J}_{\mathrm{BX}}=11.0 \mathrm{~Hz}, \mathrm{~J}_{\mathrm{AB}}=3.8 \mathrm{~Hz}, 3 \mathrm{H}, \mathrm{CHCH}_{2}\right), 2.41\left(\mathrm{~m}, 2 \mathrm{H}, \mathrm{CH}_{2}\right), 2.45,3.02(\mathrm{AB}$ system, J=18.4 Hz, $\left.2 \mathrm{H}, \mathrm{CH}_{2}\right), 2.56(\mathrm{~s}, \mathrm{OH}), 2.82\left(\mathrm{q}, \mathrm{J}=7.2 \mathrm{~Hz}, \mathrm{CH}_{2}\right), 2.92-3.00(\mathrm{~m}, 2 \mathrm{H}$, $\mathrm{CH}_{2}, 5.0-5.16\left(\mathrm{~m}, 2 \mathrm{H}\right.$, vinylic $\left.\mathrm{CH}_{2}\right), 5.62-5.7(\mathrm{~m}, 1 \mathrm{H}$, vinylic $\mathrm{CH}), 6.85(\mathrm{~s}, \mathrm{NH}) ;{ }^{13} \mathrm{C}$ NMR $\delta 7.54\left(\mathrm{CH}_{3}\right), 8.05\left(\mathrm{CH}_{3}\right), 8.34\left(\mathrm{CH}_{3}\right), 23.83\left(\mathrm{CH}_{2}\right), 30.98\left(\mathrm{CH}_{2}\right), 34.79\left(\mathrm{CH}_{2}\right)$, $38.57\left(\mathrm{CH}_{2}\right), 51.55\left(\mathrm{CH}_{2}\right), 57.24\left(\mathrm{CH}_{2}\right), 57.24(\mathrm{CH}), 60.33\left(\mathrm{CH}_{2}\right), 73.43(\mathrm{C}), 74.36(\mathrm{C})$, $118.53\left(\mathrm{CH}_{2}\right), \quad 134.22(\mathrm{CH}), \quad 153.49(\mathrm{CN}), \quad 197.78(\mathrm{CO}), \quad 212.2(\mathrm{CO}) ;$ MS $\mathrm{m} / \mathrm{z}(\%)$ : $335\left(\mathrm{M}^{+}, 29\right), 206(17), 198(24), 194(29), 154(60), 138(26), 84(100), 70(84), 57(68)$; HRMS: Calcd for $\mathrm{C}_{18} \mathrm{H}_{29} \mathrm{~N}_{3} \mathrm{O}_{3}: 335.2202$, Found: 335.2202 .

3,9-Diacetyl-7-(3-butenyl)-10-hydroxy-10-methyl-1,2,7-triazaspiro[4.5]dec-2ene(3h). $36 \%$ yield. Yellow crystal, m.p. $184-186{ }^{\circ} \mathrm{C}\left(\mathrm{Et}_{2} \mathrm{O}\right)$; ${ }^{1} \mathrm{H}$ NMR $\delta 1.08(\mathrm{~s}, 3 \mathrm{H}$, $\mathrm{CH}_{3}$ ), 2.05, 2.69(AB system, J=11.9 Hz, 2H, $\mathrm{CH}_{2}$ ), 2.46, 2.75, 2.81(ABX, J $\mathrm{AX}=12.0$ $\left.\mathrm{Hz}, \mathrm{J}_{\mathrm{BX}}=11.0 \mathrm{~Hz}, \mathrm{~J}_{\mathrm{AB}}=4.0 \mathrm{~Hz}, 3 \mathrm{H}, \mathrm{CHCH}_{2}\right), 2.20\left(\mathrm{t}, \mathrm{J}=6.4 \mathrm{~Hz}, 2 \mathrm{H}, \mathrm{CH}_{2}\right), 2.33(\mathrm{~s}, 3 \mathrm{H}$, $\mathrm{CH}_{3}$ ), 2.46, 3.01(AB system, $\left.\mathrm{J}=17.4 \mathrm{~Hz}, 2 \mathrm{H}, \mathrm{CH}_{2}\right), 2.43\left(\mathrm{~s}, 3 \mathrm{H}, \mathrm{CH}_{3}\right), 2.30-2.50(\mathrm{~m}$, $\left.2 \mathrm{H}, \mathrm{CH}_{2}\right), 2.62(\mathrm{~s}, \mathrm{OH}), 4.97-5.10\left(\mathrm{~m}, 2 \mathrm{H}\right.$, vinylic $\left.\mathrm{AB} \mathrm{CH}_{2}\right), 5.64-5.84(\mathrm{~m}, 1 \mathrm{H}$, vinylic $\mathrm{CH}), 6.86(\mathrm{~s}, \mathrm{NH}) ;{ }^{13} \mathrm{C}$ NMR $\delta$ 17.5( $\left(\mathrm{CH}_{3}\right), 25.4\left(\mathrm{CH}_{3}\right), 31.3\left(\mathrm{CH}_{2}\right), 33.0\left(\mathrm{CH}_{3}\right)$, 34.1 $\left(\mathrm{CH}_{2}\right), 51.9\left(\mathrm{CH}_{2}\right), 56.1\left(\mathrm{CH}_{2}\right), 57.3\left(\mathrm{CH}_{2}\right), 58.2(\mathrm{CH}), 71.8(\mathrm{CO}), 73.7(\mathrm{CO}), 116.0$ $\left(\mathrm{CH}_{2}\right), 136.1(\mathrm{CH}), 154.0(\mathrm{CN}), 194.8(\mathrm{CO}), 209.3(\mathrm{CO}) ; \mathrm{MS} \mathrm{m} / \mathrm{z}(\%) 307\left(\mathrm{M}^{+}, 3\right)$, 267(29), 266(100), 248(13), 205(12), 193(6), 178(28), 154(14), 123 (14), 84(34), 71(27), 43(54); HRMS Calcd for $\mathrm{C}_{16} \mathrm{H}_{25} \mathrm{~N}_{3} \mathrm{O}_{3}: 307.1890$, Found:307.1889.

10-Ethyl-7-(2-butenyl)-10-hydroxy-3,9-Dipropionyl-1,2,7-triazaspiro[4.5]dec-2ene(3i): White crystal, 34\%, m.p. $148-150^{\circ} \mathrm{C}\left(\mathrm{Et}_{2} \mathrm{O}\right)$; ${ }^{1} \mathrm{H} \mathrm{NMR}-\delta 0.75(\mathrm{t}, \mathrm{J}=15 \mathrm{~Hz}$, $\left.3 \mathrm{H}, \mathrm{CH}_{3}\right), 0.98\left(\mathrm{t}, \mathrm{J}=14.3 \mathrm{~Hz}, 3 \mathrm{H}, \mathrm{CH}_{3}\right), 1.09\left(\mathrm{t}, \mathrm{J}=14.8 \mathrm{~Hz}, 3 \mathrm{H}, \mathrm{CH}_{3}\right), 1.24-1.43$, $1.69-1.78\left(\mathrm{~m}, 2 \mathrm{H}, \mathrm{CH}_{2}\right), 1.62\left(\mathrm{~d}, \mathrm{~J}=16.8 \mathrm{~Hz}, 3 \mathrm{H}, \mathrm{CH}_{3}\right), 1.96,2.65(\mathrm{AB}$ system, $2 \mathrm{H}$, $\left.\mathrm{CH}_{2}\right), 2.30-2.40\left(\mathrm{~m}, 2 \mathrm{H}, \mathrm{CH}_{2}\right), 2.40,2.94,3.04(\mathrm{ABX}, \mathrm{J}=7.0 \mathrm{~Hz}, \mathrm{~J}=7.0 \mathrm{~Hz}, 2 \mathrm{H}$, $\mathrm{CH}_{2}$ ), 3H, $\left.\mathrm{CHCH}_{2}\right), 2.42,3.00\left(\mathrm{AB}\right.$ system, $\left.\mathrm{J}=18.0 \mathrm{~Hz}, 2 \mathrm{H}, \mathrm{CH}_{2}\right), 2.50(\mathrm{~s}, \mathrm{OH}), 2.81(\mathrm{q}$, $\left.\mathrm{J}=7.2 \mathrm{~Hz}, 2 \mathrm{H}, \mathrm{CH}_{2}\right), 2.88\left(\mathrm{~d}, \mathrm{~J}=6.4 \mathrm{~Hz}, 2 \mathrm{H}, \mathrm{CH}_{2}\right), 5.33-5.35(\mathrm{~m}, 1 \mathrm{H}$, olefinic $\mathrm{H})$, 5.47-5.48(m, $1 \mathrm{H}$, olefinic $\mathrm{H}), 6.88(\mathrm{~s}, \mathrm{NH}) ;{ }^{13} \mathrm{C} \mathrm{NMR} \delta 7.55\left(\mathrm{CH}_{3}\right), 8.08\left(\mathrm{CH}_{3}\right)$, 8.35 $\left(\mathrm{CH}_{3}\right), \quad 17.75\left(\mathrm{CH}_{3}\right), \quad 23.83\left(\mathrm{CH}_{2}\right), 30.99\left(\mathrm{CH}_{2}\right), \quad 34.82\left(\mathrm{CH}_{2}\right), \quad 38.59\left(\mathrm{CH}_{2}\right)$, 51.32( $\left(\mathrm{CH}_{2}\right), 57.26(\mathrm{CH}), 57.36\left(\mathrm{CH}_{2}\right), 59.61\left(\mathrm{CH}_{2}\right), 73.52(\mathrm{C}), 74.4(\mathrm{C}), 126.78(\mathrm{CH})$,

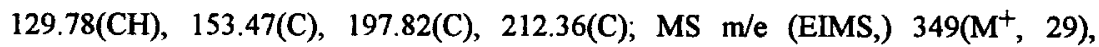
292(16), 212(32), 208(20), 191(22), 169(21), 168(57), 138(22), 137(27), 110(31), 98(100), 57(64), 55(74); HRMS: Calcd for $\mathrm{C}_{19} \mathrm{H}_{31} \mathrm{~N}_{3} \mathrm{O}_{3}: 349.2538$, Found 349.2360 . 
Acknowledgements: This work was supported by the National Science Council of the Republic of China.

References and Notes:

1. Broeckx,W.,Overbergh, N., Samyn,C. Smets, G., and L'abbe, G., Tetrahedron, 1971, 27, 3527-3534.

2. Yang, C. H. and Shen, H. J., Tetrahedron Lett., 1993, 34, 4051-4054.

3. Spiro compounds consisting of 1- or 2-pyrazolines and cyclohexanone, pyran-4one or thiopyran-4-one have been reported; (a). Toth, G, Levai, A. and Duddeck, H., Mag. Reson. Chem., 1992, 30, 235-239. (b). Toth, G., Levai, A., Szollosy, A. and Duddeck, H., Tetrahedron, 1993, 49, 863-880.

4. Posner, G.H., Chem. Rev., 1986, 86, 831-844.

5. Posner, G. H., Mallamo, J. P., and Black, A. Y., Tetrahedron, 1981, 37, 39213926.

6. Grotjahn, D. B. and Vollhardt, K. P. C., J. Am. Chem. Soc., 1986, 108, 20912093.

7. Martin, G. E. and Crouch, R. C., J. Nat. Prod., 1991, 54, 1-70.

8. $3 \mathrm{a}, 3 \mathrm{~b}$, and $3 \mathrm{~g}$ are by-products from the reactions of allyl azide or 2-butenyl azide with alkyl vinyl ketone. The major products therein are 2 . The yields of these by-products are in the range of $0-25 \%$ dependent on the relative concentraction of the alkyl vinyl ketone to that of azide.

9. Gabe, E. J., Le Page, Y., Charland, J. P., Lee, F. L., and White, P. S., $J$. Appl. Cryst. 1989, 22, 384-387. 